Supplement of Clim. Past, 16, 245-263, 2020

https://doi.org/10.5194/cp-16-245-2020-supplement

(c) Author(s) 2020. This work is distributed under

the Creative Commons Attribution 4.0 License.

(c) (1)

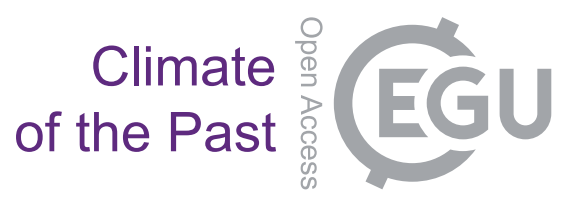

Supplement of

\title{
Algal lipids reveal unprecedented warming rates in alpine areas of SW Europe during the industrial period
}

Antonio García-Alix et al.

Correspondence to: Antonio García-Alix (agalix@ugr.es)

The copyright of individual parts of the supplement might differ from the CC BY 4.0 License. 

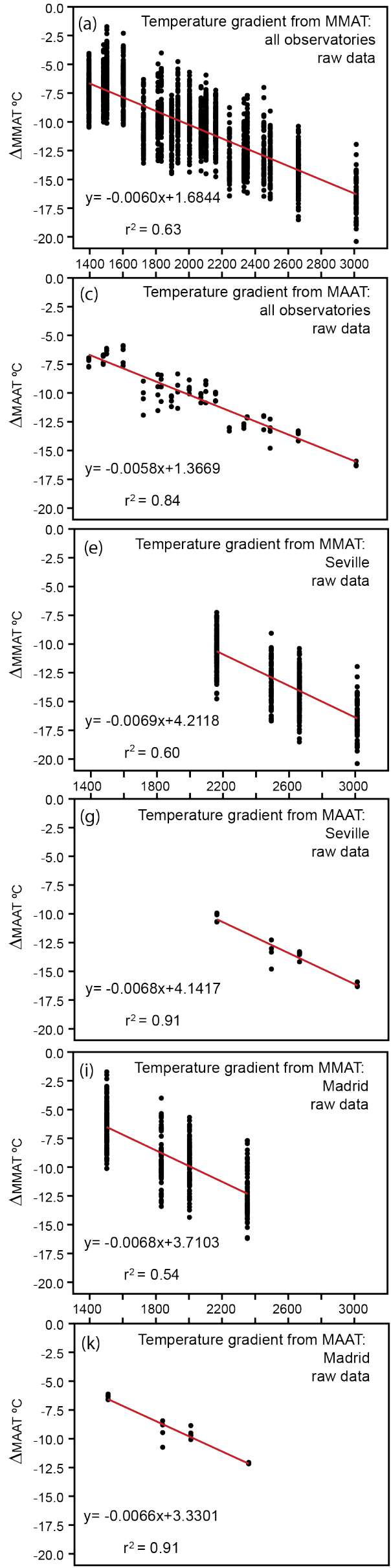
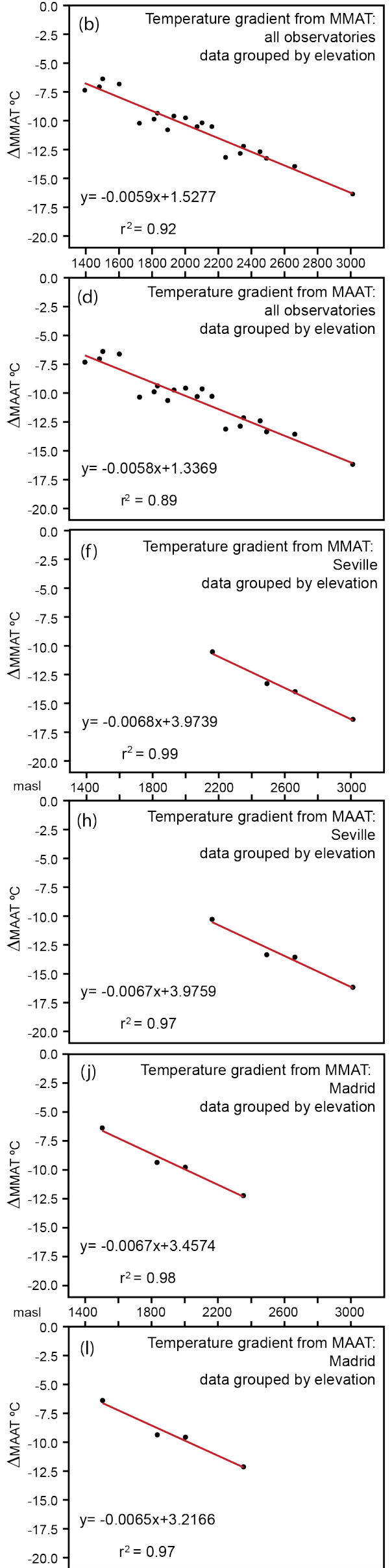
Supplementary Figure S1. Calculation of the Environmental Lapse Rate (ELR, $\left.{ }^{\circ} \mathrm{C} / \mathrm{m}\right)$ by means of ordinary least square regressions from temperature and elevation variations ( $\Delta_{\text {elevation }}$ and $\Delta$ MMAT or $\Delta_{\text {MAAT}}$ ) between the low and high elevation observatories listed in Table S1 (data from Gonzalez-Hidalgo et al., 2015; Observatorio del cambio global de Sierra Nevada, 2019; Spanish National Weather Agency - AEMet Open Data, 2019; Staudt et al., 2007). MMAT (Monthly Mean Air Temperature) MAAT (Mean Annual

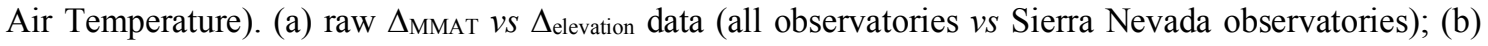

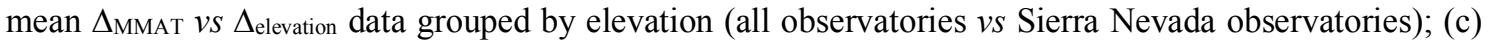
raw $\Delta_{\text {MAAT }} v s \Delta_{\text {elevation }}$ data (all observatories $v s$ Sierra Nevada observatories); (d) mean $\Delta_{\text {MAAT }} v s \Delta_{\text {elevation }}$ data grouped by elevation (all observatories $v s$ Sierra Nevada observatories); (e) raw $\Delta_{\text {MMAT }} v s \Delta_{\text {elevation }}$ (Sevilla observatory vs Sierra Nevada observatories); (f) mean $\Delta_{\text {MмAт }} v s \Delta_{\text {elevation }}$ (mean data grouped by elevation: Sevilla observatory $v s$ Sierra Nevada observatories); (g) raw $\Delta_{\text {MAAT }} v s \Delta_{\text {elevation }}$ (Sevilla observatory $v s$ Sierra Nevada observatories); (h) mean $\Delta$ MAAT $v s \Delta_{\text {elevation }}$ (mean data grouped by elevation: Sevilla observatory $v s$ Sierra Nevada observatories); (i) raw $\Delta$ MMAT $v s$ elevation from (Madrid observatory vs Sierra Nevada observatories); (j) mean $\Delta$ ммат $v s \Delta_{\text {elevation }}$ (mean data grouped by elevation: Madrid observatory $v s$ Sierra Nevada observatories); (k) raw $\Delta_{\text {MAAT }} v s \Delta_{\text {elevation }}$ (Madrid observatory $v s$ Sierra Nevada observatories); (1) mean $\Delta_{\text {MAAT }} v s \Delta_{\text {elevation }}$ (mean data grouped by elevation: Madrid observatory $v s$ Sierra Nevada observatories). The obtained ERLs (ranging from $0.0058^{\circ} \mathrm{C} / \mathrm{m}$ to $0.0069^{\circ} \mathrm{C} / \mathrm{m}$ ) are certainly close to the global mean ERL $\left(\sim 0.0065^{\circ} \mathrm{C} / \mathrm{m}\right)$ (International Civil Aviation Organization, 1993), showing that the different calculations are in agreement with the global temperature-elevation gradients. 


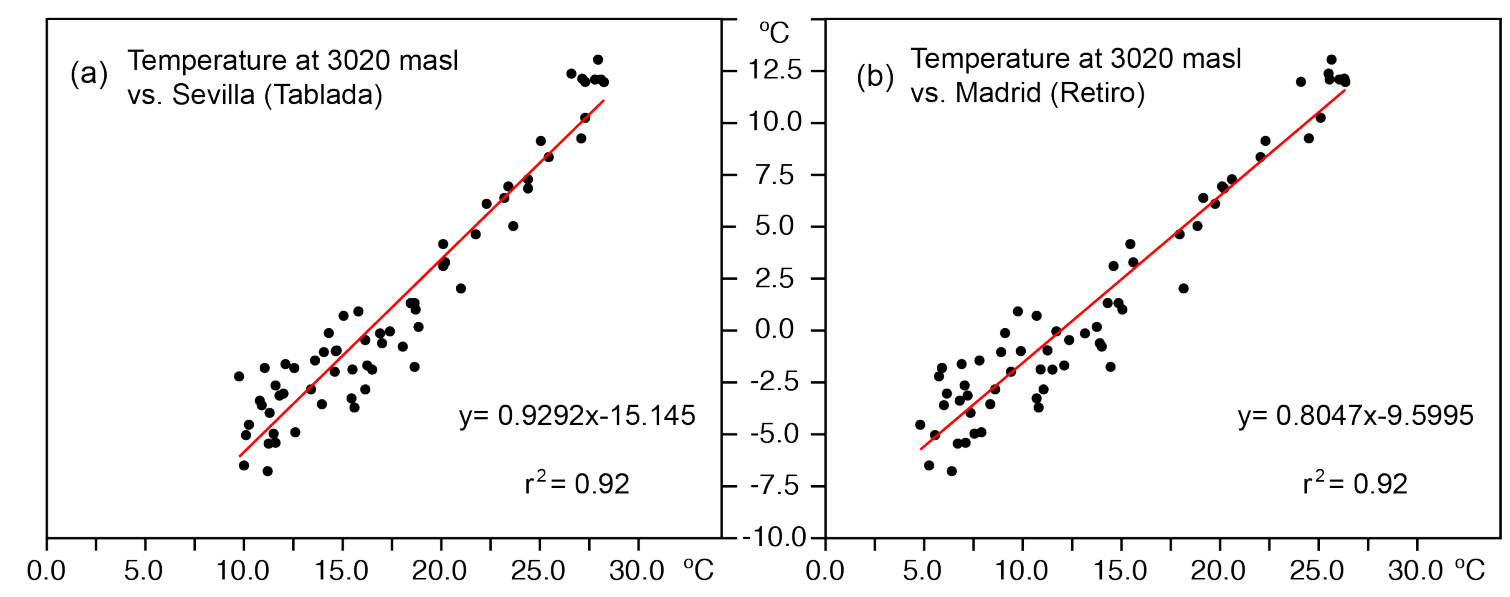

Supplementary Figure S2. (a) Correlation by means of ordinary least square regression between Sevilla monthly air temperatures and those from Cetursa 5 observatory (3020 masl). (b) Correlations by means of ordinary least square regression between Madrid monthly air temperatures and those from Cetursa 5 observatory (3020 masl) (data from Gonzalez-Hidalgo et al., 2015; Observatorio del cambio global de Sierra Nevada, 2019; Spanish National Weather Agency - AEMet Open Data, 2019; Staudt et al., 2007). 
LDI vs. reference temperature time-series 1 at 3020 masl from 1908 to 2008

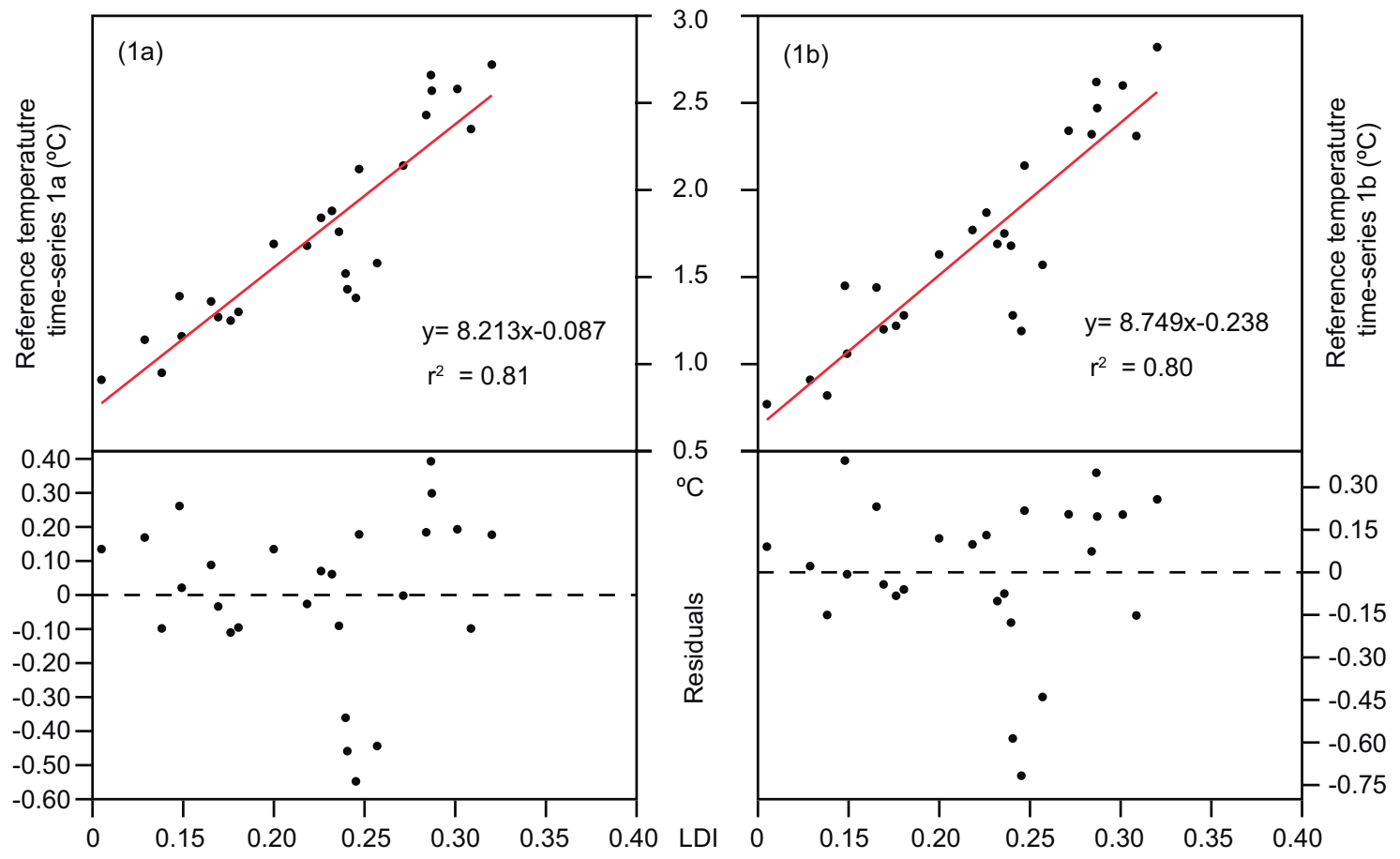

LDI vs. reference temperature time-series 2 at 3020 masl from 1908 to 2008

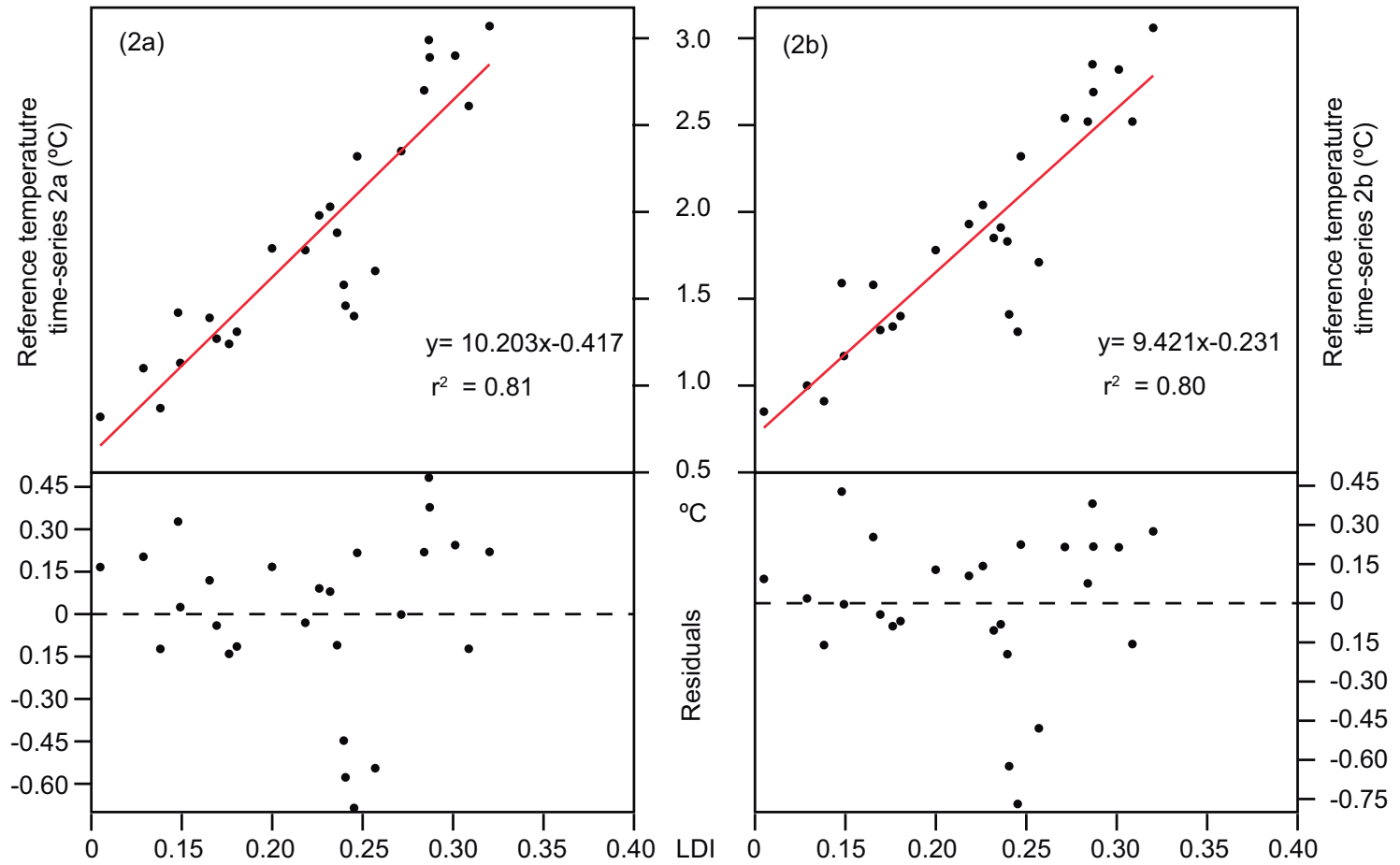

Supplementary Figure S3. LDI-temperature calibraton (ordinary least square regression) for each of the reference temperture time-series at 3020 masl from 1908 to 2008.

$\begin{array}{ll}\text { LDI calibration 1a: MAAT }=8.213 * \text { LDI-0.087 } & r^{2}=0.81 \\ \text { LDI calibration 1b: MAAT }=8.749 * \text { LDI-0.238 } & r^{2}=0.80 \\ \text { LDI calibration 2a: MAAT }=10.203 * \text { LDI- } 0.417 & r^{2}=0.81 \\ \text { LDI calibration 2b: MAAT }=9.421 * \text { LDI- } 0.231 & r^{2}=0.80\end{array}$ 

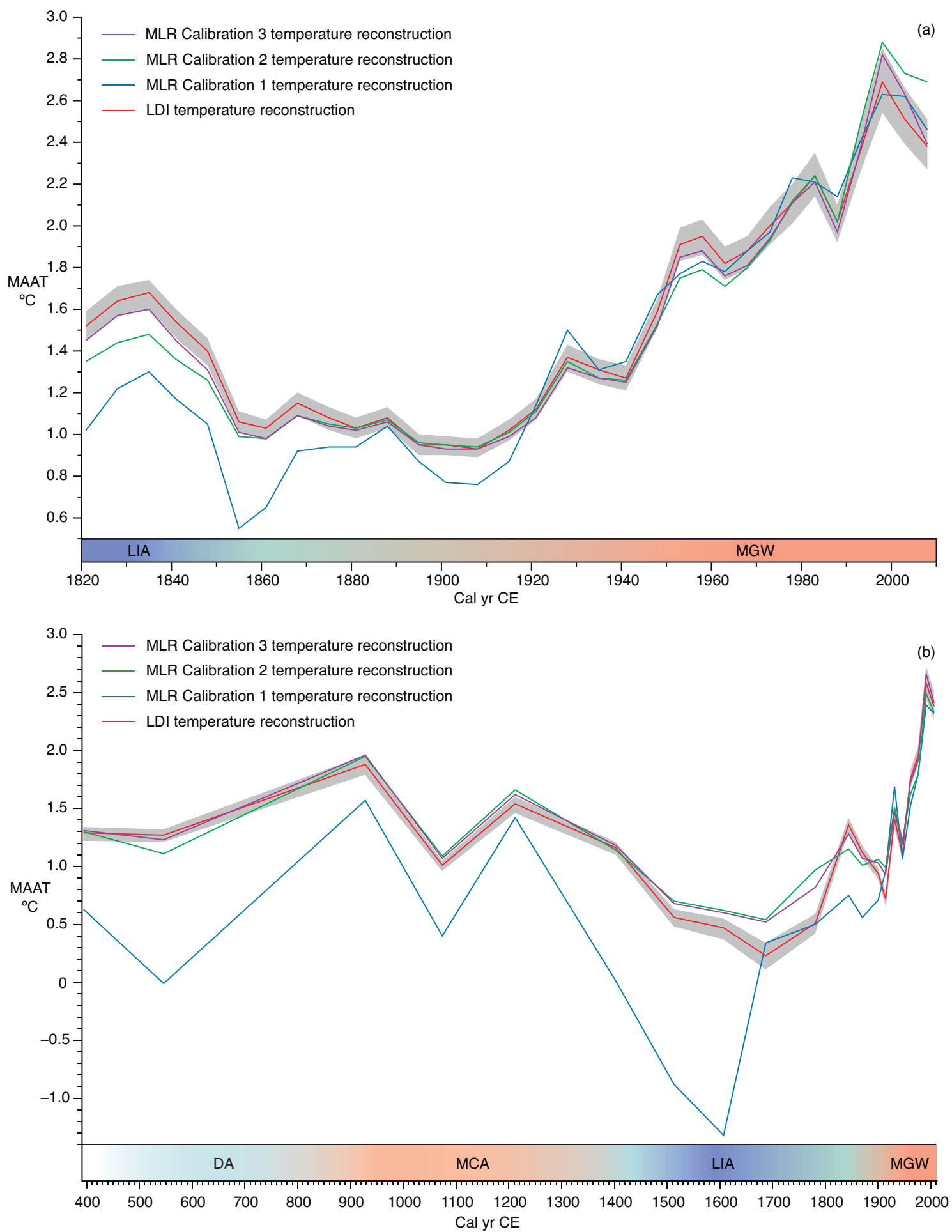

Supplementary Figure S4. Comparison between the reconstructed mean annual air temperatures (MAAT) from both short and long LdRS records obtained from the different LCD-based temperature calibrations: 1) the LDI temperature calibration (Eq. 2: Fig. 3) along with its maximum and minimum range of vatiation from individual LDI-calibrations (grey shade), and 2) the MLR calibrations 1, 2 and 3 (Table S8). (a) Short core data (last $\sim 200$ years). (b) Long core data (last $\sim 1500$ years). 


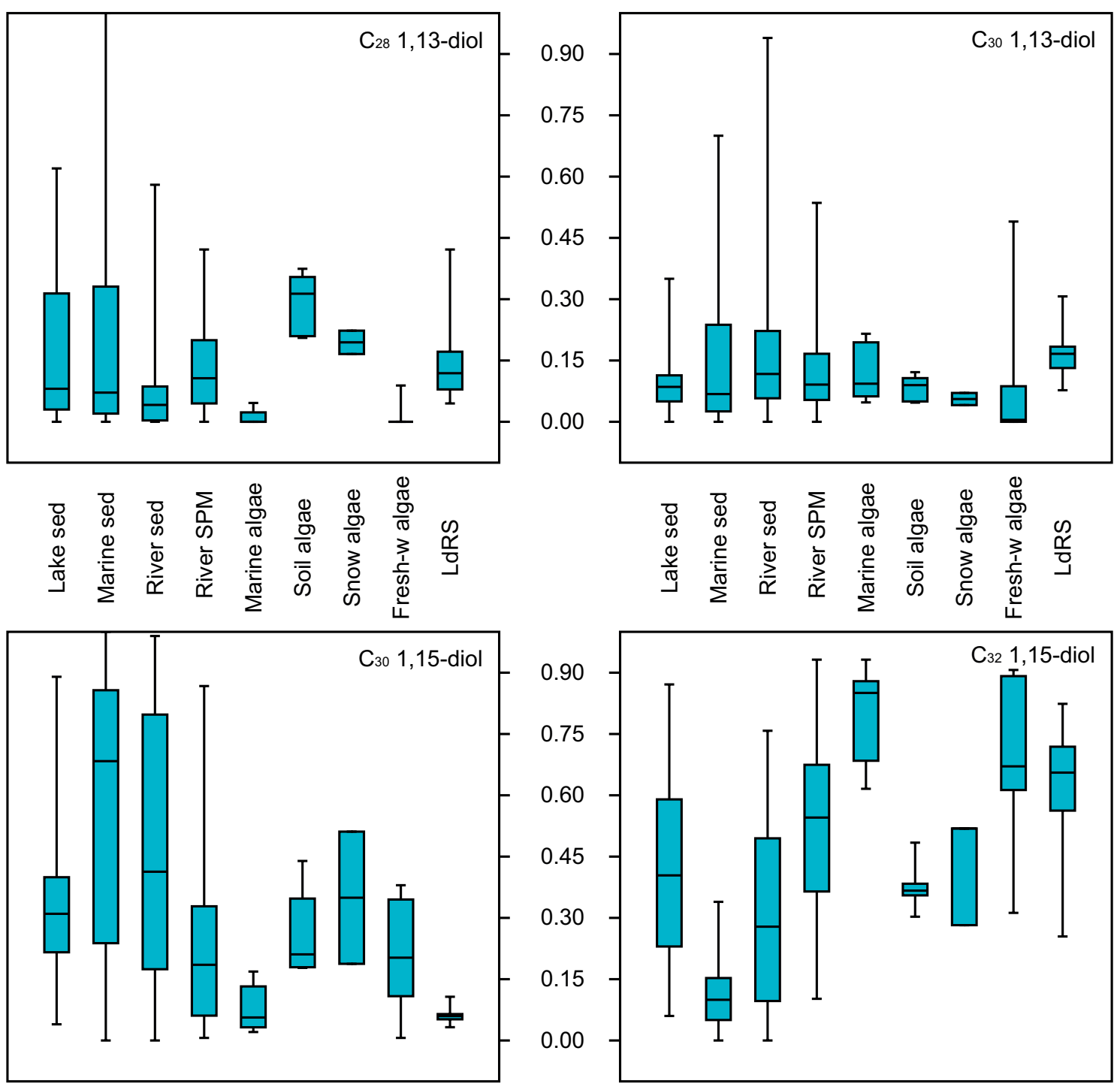

Supplementary Figure S5. Comparison by means of box-plot diagrams of the distribution of the relative abundances of the main LCDs from different sources and those form LdRS. Literature data from lake sediments (Rampen et al., 2014a), algal cultures (Rampen et al., 2014a), marine sediments (de Bar et al., 2016; Lattaud et al., 2017a; Rampen et al., 2014b; Rampen et al., 2012), river sediments/inputs (de Bar et al., 2016; Lattaud et al., 2017b), river particulate organic matter (Lattaud et al., 2018). 


\begin{tabular}{|l|c|l|c|c|}
\hline Observatory & $\begin{array}{c}\text { Observatory } \\
\text { elevation } \\
\text { (masl) }\end{array}$ & $\begin{array}{c}\text { Sierra Nevada (SN) } \\
\text { observatory }\end{array}$ & $\begin{array}{c}\text { SN } \\
\text { Observatory } \\
\text { elevation } \\
\text { (masl) }\end{array}$ & $\begin{array}{c}\Delta_{\text {elevation }} \\
\text { (masl) }\end{array}$ \\
\hline Sevilla (Tablada) & 8 & Albergue & 2500 & 2492 \\
\hline Sevilla (Tablada) & 8 & Cetursa 1 & 2170 & 2162 \\
\hline Sevilla (Tablada) & 8 & Cetursa 3 & 2670 & 2662 \\
\hline Sevilla (Tablada) & 8 & Cetursa 5 & 3020 & 3012 \\
\hline Granada Airport & 567 & Albergue & 2500 & 1933 \\
\hline Granada Airport & 567 & Cetursa 1 & 2170 & 1603 \\
\hline Granada Airport & 567 & Cetursa 3 & 2670 & 2103 \\
\hline Granada Airport & 567 & Cetursa 5 & 3020 & 2453 \\
\hline Madrid (Retiro) & 667 & Albergue & 2500 & 1733 \\
\hline Madrid (Retiro) & 667 & Cetursa 1 & 2670 & 2003 \\
\hline Madrid (Retiro) & 667 & Cetursa 3 & 3020 & 2353 \\
\hline Madrid (Retiro) & 667 & Cetursa 5 & 2500 & 1813 \\
\hline Granada Armilla & 687 & Albergue & 2170 & 1483 \\
\hline Granada Armilla & 687 & Cetursa 1 & 2670 & 1983 \\
\hline Granada Armilla & 687 & Cetursa 3 & 3020 & 2333 \\
\hline Granada Armilla & 687 & Cetursa 5 & 2500 & 1725 \\
\hline Granada Cartuja & 775 & Albergue & 170 & 1395 \\
\hline Granada Cartuja & 775 & Cetursa 1 & Cetursa 3 & 2245 \\
\hline Granada Cartuja & 775 & Cetursa 5 & & 1503 \\
\hline Granada Cartuja & 775 & & 2670 & \\
\hline
\end{tabular}

Supplementary Table S1. Elevational difference between low and high elevation observatories used in this study. Observatories: Granada city ( $\sim 30 \mathrm{~km}$ from LdRS), Madrid ( $\sim 360 \mathrm{~km}$ from LdRS) and Sevilla ( $230 \mathrm{~km}$ from LdRS but almost similar latitude as LdRS). 


\begin{tabular}{|c|c|c|c|c|c|c|c|c|}
\hline \multirow{2}{*}{$\begin{array}{l}\text { Low vs high elevation } \\
\text { observatories }\end{array}$} & \multicolumn{2}{|c|}{$\begin{array}{c}\text { Cetursa } 53020 \text { masl } \\
(n=67)\end{array}$} & \multicolumn{2}{|c|}{$\begin{array}{c}\text { Cetursa } 32670 \text { masl } \\
(n=113)\end{array}$} & \multicolumn{2}{|c|}{$\begin{array}{c}\text { Cetursa } 12170 \text { masl } \\
(n=121)\end{array}$} & \multicolumn{2}{|c|}{$\begin{array}{l}\text { Albergue } 2500 \text { masl } \\
\qquad(n=72)\end{array}$} \\
\hline & $r$ & $p$ & $r$ & $p$ & $r$ & $p$ & $r$ & $p$ \\
\hline Madrid 667 masl & 0.95920 & 7.99E-37 & 0.9671 & $8.28 \mathrm{E}-68$ & 0.9641 & $2.44 \mathrm{E}-70$ & 0.9598 & $2.40 \mathrm{E}-40$ \\
\hline Sevilla 8 masl & 0.95790 & $2.14 \mathrm{E}-36$ & 0.9635 & $2.24 \mathrm{E}-65$ & 0.9620 & $5.85 \mathrm{E}-69$ & 0.9641 & $4.86 \mathrm{E}-42$ \\
\hline Gr-Airport 567 masl & 0.95430 & $2.80 \mathrm{E}-35$ & 0.9655 & 4.18E-66 & 0.9626 & $8.66 \mathrm{E}-69$ & 0.9591 & 4.37E-40 \\
\hline Gr-Cartuja 775 masl & 0.96887 & $1.61 \mathrm{E}-40$ & 0.9769 & $3.39 \mathrm{E}-76$ & 0.9751 & $1.04 \mathrm{E}-79$ & 0.9680 & $9.63 \mathrm{E}-44$ \\
\hline Gr-Armilla 687 masl & 0.96821 & $3.11 \mathrm{E}-40$ & 0.9783 & $9.84 \mathrm{E}-78$ & 0.9767 & $2.01 \mathrm{E}-81$ & 0.9645 & $3.22 \mathrm{E}-42$ \\
\hline
\end{tabular}

Supplementary Table S2. Pearson correlations between mean monthly air temperatures (MMAT) from low and high elevation (Sierra Nevada) observatories (data from Gonzalez-Hidalgo et al., 2015; Observatorio del cambio global de Sierra Nevada, 2019; Spanish National Weather Agency - AEMet Open Data, 2019; Staudt et al., 2007). The Mann-Kendall test performed using PAST software (Hammer et al., 2001 ) in the raw data of these variables showed a $p>0.05$ pointing towards no linear trends; therefore, no transformation has been applied. 


\begin{tabular}{|c|c|c|c|c|c|c|c|c|c|c|c|}
\hline \multirow{2}{*}{$\begin{array}{c}\text { LDI LdRS she } \\
\text { vs Low } \\
\text { elevation } \\
\text { observatories } \\
\end{array}$} & \multirow{2}{*}{$\mathbf{n}$} & \multicolumn{2}{|c|}{ LDI } & \multicolumn{2}{|c|}{$\mathrm{C}_{28}$ 1,13-diol } & \multicolumn{2}{|c|}{$\mathrm{C}_{30}$ 1,15-diol } & \multicolumn{2}{|c|}{$\mathrm{C}_{30}$ 1,13-diol } & \multicolumn{2}{|c|}{$\mathrm{C}_{32}$ 1,15-diol } \\
\hline & & $r$ & $p$ & $r$ & $p$ & $r$ & $p$ & $r$ & $p$ & $r$ & $p$ \\
\hline Sevilla MAAT & 19 & 0.91 & $6.6 \mathrm{E}-08$ & -0.86 & $2.4 \mathrm{E}-06$ & 0.41 & 0.081 & -0.91 & $6.0 \mathrm{E}-08$ & 0.89 & 4.1E-07 \\
\hline Sevilla MWAT & 19 & 0.72 & $5.6 \mathrm{E}-04$ & -0.70 & $9.5 \mathrm{E}-04$ & 0.09 & 0.718 & -0.75 & 2.3E-04 & 0.74 & $3.2 \mathrm{E}-04$ \\
\hline Madrid MAAT & 19 & 0.91 & 8.2E-08 & -0.87 & $1.6 \mathrm{E}-06$ & 0.34 & 0.155 & -0.92 & $2.6 \mathrm{E}-08$ & 0.90 & $1.5 \mathrm{E}-07$ \\
\hline Madrid MWAT & 19 & 0.77 & $1.3 \mathrm{E}-04$ & -0.76 & $1.4 \mathrm{E}-04$ & 0.15 & 0.541 & -0.82 & $1.8 \mathrm{E}-05$ & 0.81 & $2.9 \mathrm{E}-05$ \\
\hline Gr-Airport MAAT & 19 & 0.71 & 7.2E-04 & -0.62 & $5.0 \mathrm{E}-03$ & 0.32 & 0.184 & -0.72 & $5.1 \mathrm{E}-04$ & 0.65 & 2.3E-03 \\
\hline Gr-Airport MWAT & 19 & 0.58 & $9.5 \mathrm{E}-03$ & -0.56 & $1.3 \mathrm{E}-02$ & 0.07 & 0.772 & -0.63 & $4.0 \mathrm{E}-03$ & 0.60 & 7.1E-03 \\
\hline Gr-Cartuja MAAT & 19 & 0.73 & 3.7E-04 & -0.70 & $9.2 \mathrm{E}-04$ & 0.29 & 0.222 & -0.76 & $1.5 \mathrm{E}-04$ & 0.73 & 4.4E-04 \\
\hline Gr-Cartuja MWAT & 19 & 0.65 & $2.8 \mathrm{E}-03$ & -0.65 & $2.8 \mathrm{E}-03$ & 0.13 & 0.600 & -0.70 & $8.6 \mathrm{E}-04$ & 0.68 & $1.4 \mathrm{E}-03$ \\
\hline Gr-Armilla MAAT & 19 & 0.70 & $9.1 \mathrm{E}-04$ & -0.68 & $1.3 \mathrm{E}-03$ & 0.31 & 0.203 & -0.73 & $3.6 \mathrm{E}-04$ & 0.70 & $7.5 \mathrm{E}-04$ \\
\hline Gr-Armilla MWAT & 19 & 0.61 & $5.6 \mathrm{E}-03$ & -0.64 & $2.9 \mathrm{E}-03$ & 0.18 & 0.456 & -0.66 & $2.0 \mathrm{E}-03$ & 0.66 & 2.3E-03 \\
\hline \multirow{2}{*}{$\begin{array}{c}\text { LDI LdRS she +lgc } \\
\text { vs Low } \\
\text { elevation } \\
\text { observatories }\end{array}$} & \multirow[b]{2}{*}{$\mathbf{n}$} & \multicolumn{2}{|c|}{ LDI } & \multicolumn{2}{|c|}{$\mathrm{C}_{28}$ 1,13-diol } & \multicolumn{2}{|c|}{$\mathrm{C}_{30}$ 1,15-diol } & \multicolumn{2}{|c|}{$\mathrm{C}_{30}$ 1,13-diol } & \multicolumn{2}{|c|}{$\mathrm{C}_{32}$ 1,15-diol } \\
\hline & & $r$ & $p$ & $r$ & $p$ & $r$ & $p$ & $r$ & $p$ & $r$ & $p$ \\
\hline Sevilla MAAT & 26 & 0.89 & $7.5 \mathrm{E}-10$ & -0.72 & $3.9 \mathrm{E}-05$ & 0.34 & 0.0855 & -0.86 & $1.4 \mathrm{E}-08$ & 0.79 & $1.8 \mathrm{E}-06$ \\
\hline Sevilla MWAT & 26 & 0.77 & 4.7E-06 & -0.65 & $2.9 \mathrm{E}-04$ & 0.20 & 0.3360 & -0.74 & $1.5 \mathrm{E}-05$ & 0.71 & 4.3E-05 \\
\hline Madrid MAAT & 26 & 0.90 & $2.9 \mathrm{E}-10$ & -0.73 & $2.3 \mathrm{E}-05$ & 0.30 & 0.1335 & -0.88 & 2.7E-09 & 0.81 & 5.7E-07 \\
\hline Madrid MWAT & 26 & 0.80 & $1.0 \mathrm{E}-06$ & -0.68 & $1.3 \mathrm{E}-04$ & 0.22 & 0.2886 & -0.79 & $1.5 \mathrm{E}-06$ & 0.75 & $1.1 \mathrm{E}-05$ \\
\hline Gr-Airport MAAT & 26 & 0.69 & $9.5 \mathrm{E}-05$ & -0.55 & $3.4 \mathrm{E}-03$ & 0.27 & 0.1748 & -0.67 & $2.0 \mathrm{E}-04$ & 0.61 & $1.0 \mathrm{E}-03$ \\
\hline Gr-Airport MWAT & 26 & 0.62 & $6.8 \mathrm{E}-04$ & -0.54 & 4.1E-03 & 0.19 & 0.3636 & -0.61 & $1.1 \mathrm{E}-03$ & 0.59 & $1.6 \mathrm{E}-03$ \\
\hline Gr-Cartuja MAAT & 26 & 0.74 & $1.4 \mathrm{E}-05$ & -0.65 & $3.5 \mathrm{E}-04$ & 0.26 & 0.2075 & -0.74 & $1.8 \mathrm{E}-05$ & 0.70 & $6.8 \mathrm{E}-05$ \\
\hline Gr-Cartuja MWAT & 26 & 0.71 & $4.5 \mathrm{E}-05$ & -0.67 & $2.1 \mathrm{E}-04$ & 0.23 & 0.2685 & -0.69 & $8.5 \mathrm{E}-05$ & 0.70 & $7.1 \mathrm{E}-05$ \\
\hline Gr-Armilla MAAT & 26 & 0.69 & $1.1 \mathrm{E}-04$ & -0.59 & $1.6 \mathrm{E}-03$ & 0.25 & 0.2102 & -0.68 & $1.2 \mathrm{E}-04$ & 0.64 & 4.4E-04 \\
\hline Gr-Armilla MWAT & 26 & 0.65 & $3.0 \mathrm{E}-04$ & -0.60 & $1.2 \mathrm{E}-03$ & 0.24 & 0.2380 & -0.64 & $4.8 \mathrm{E}-04$ & 0.63 & $5.6 \mathrm{E}-04$ \\
\hline
\end{tabular}

Supplementary Table S3. Pearson correlations for the last $\sim 100$ years between LDI data in LdRS and temperature time-series of different observatories. Upper part: LDI LdRS she data; lower part: LDI LdRS shc $+\operatorname{lgc}$ data. Mean annual air temperature (MAAT) and mean seasonal air temperatures from the warmer months (May-September: MWAT) have been tested (data from Gonzalez-Hidalgo et al., 2015; Spanish National Weather Agency - AEMet Open Data, 2019; Staudt et al., 2007). Although the reconstructed temperatures from Granada for the last $\sim 100$ years show a good correlation with the LCD distributions, they are likely biased by the low quality of the reconstructed data, since there are only reliable data from the 1970s onwards. In addition to the quality of the records (presence of gaps), this discrepancy between the LCD distribution in LdRS and Granada time-series could be linked to the strong control of Granada basin geomorphology in local rain and temperature patterns at low elevations, with local clouds and specific microclimate. Madrid and Sevilla area would not be influenced by those effects (Dogniaux, 1994). 


\begin{tabular}{|c|c|c|c|c|c|c|c|c|}
\hline \multirow{2}{*}{$\begin{array}{c}\text { LDI LdRS she } \\
\text { vs Low } \\
\text { elevation } \\
\text { observatories }\end{array}$} & \multirow{2}{*}{$\mathbf{n}$} & \multicolumn{2}{|c|}{ Normal correlation } & \multicolumn{2}{|c|}{$\begin{array}{l}(\mathrm{z}-\mathrm{score})^{2} \\
\text { correlation }\end{array}$} & \multirow{2}{*}{$\begin{array}{c}\text { Mann- } \\
\text { Kendall } \\
\text { no trend } \\
p>0.05 \\
\end{array}$} & \multicolumn{2}{|c|}{ Detrended correlation } \\
\hline & & $r$ & $p$ & $r$ & $p$ & & $r$ & $p$ \\
\hline Sevilla MAAT & 19 & 0.91 & $7 \mathrm{E}-08$ & 0.89 & $2 \mathrm{E}-07$ & 0.63 & 0.95 & $9 \mathrm{E}-10$ \\
\hline Sevilla MWAT & 19 & 0.72 & 0.0005 & 0.27 & 0.2719 & 0.04 & 0.40 & 0.0935 \\
\hline Madrid MAAT & 19 & 0.91 & $8 \mathrm{E}-08$ & 0.84 & $7 \mathrm{E}-06$ & 0.65 & 0.93 & 9E-09 \\
\hline Madrid MWAT & 19 & 0.77 & 0.0001 & 0.51 & 0.0267 & 0.09 & 0.64 & 0.0040 \\
\hline \multirow{2}{*}{$\begin{array}{c}\text { LDI LdRS she+lge } \\
\text { vs Low } \\
\text { elevation } \\
\text { observatories }\end{array}$} & \multirow{2}{*}{$\mathbf{n}$} & \multicolumn{2}{|c|}{ Normal correlation } & \multicolumn{2}{|c|}{$\begin{array}{c}{\text { (z-score })^{2}} \\
\text { correlation }\end{array}$} & $\begin{array}{l}\text { Mann- } \\
\text { Kendall }\end{array}$ & \multicolumn{2}{|c|}{ Detrended correlation } \\
\hline & & $r$ & $p$ & $r$ & $p$ & $\begin{array}{c}\text { no trend } \\
p>0.05\end{array}$ & $r$ & $p$ \\
\hline Sevilla MAAT & 26 & 0.89 & $8 \mathrm{E}-10$ & 0.81 & $6 \mathrm{E}-07$ & 0.57 & 0.86 & $1 \mathrm{E}-08$ \\
\hline Sevilla MWAT & 26 & 0.77 & $5 \mathrm{E}-06$ & 0.44 & 0.02 & 0.03 & 0.57 & 0.002 \\
\hline Madrid MAAT & 26 & 0.90 & $3 \mathrm{E}-10$ & 0.76 & $6 \mathrm{E}-06$ & 0.45 & 0.86 & $2 \mathrm{E}-08$ \\
\hline Madrid MWAT & 26 & 0.80 & $1 \mathrm{E}-06$ & 0.56 & 0.003 & 0.06 & 0.68 & 0.0001 \\
\hline
\end{tabular}

Supplementary Table S4. Pearson correlations (normal and detrended) for the last $\sim 100$ years between LDI data in LdRS and temperature time-series of different observatories. Upper part: LDI LdRS she data; lower part: LDI LdRS shc+lgc data. Normal correlations show the relationship between long-term trends (data from Gonzalez-Hidalgo et al., 2015; Spanish National Weather Agency - AEMet Open Data, 2019; Staudt et al., 2007). Data were standardised (z-scores), normalised (squares) and a Mann-Kendall trend test was performed using PAST software (Hammer et al., 2001) in order to assess the existence of any trend over time in the data series. Afterwards, data were detrended by fitting a linear regression versus time, and a Pearson correlation was calculated with the residuals. 


\begin{tabular}{|c|c|c|c|c|c|c|}
\hline $\begin{array}{c}\Delta_{\text {temperature }} \\
\text { vs } \\
\Delta_{\text {elevation }}\end{array}$ & $\begin{array}{c}\Delta_{\text {temp from }} \\
\text { eq } \boldsymbol{b} \text { (global } \\
\Delta_{\text {ММАТ) }}\end{array}$ & $\begin{array}{c}\Delta_{\text {temp }} \text { from } \\
e q \boldsymbol{d} \text { (global } \\
\Delta_{\text {МААТ }}\end{array}$ & $\begin{array}{c}\Delta_{\text {temp from } \text { eqs } f} \\
\text { and } j \text { (MМAT) }\end{array}$ & $\begin{array}{c}\Delta_{\text {temp }} \text { from eqs } \\
\boldsymbol{h} \text { and } \boldsymbol{l} \\
\text { (MAAT) }\end{array}$ & $\begin{array}{c}\text { Real } \Delta_{\text {temp }} \\
\text { from } \\
\text { MMAT }\end{array}$ & $\begin{array}{c}\text { Real } \Delta_{\text {temp }} \\
\text { from MAAT }\end{array}$ \\
\hline $\begin{array}{l}\text { Sevilla } \\
8 \text { masl }\end{array}$ & $16.24^{\circ} \mathrm{C}$ & $16.13^{\circ} \mathrm{C}$ & $16.51^{\circ} \mathrm{C}$ & $16.20^{\circ} \mathrm{C}$ & $16.39^{\circ} \mathrm{C}$ & $16.17^{\circ} \mathrm{C}$ \\
\hline $\begin{array}{l}\text { Madrid } \\
667 \text { masl }\end{array}$ & $12.36^{\circ} \mathrm{C}$ & $12.31^{\circ} \mathrm{C}$ & $12.31^{\circ} \mathrm{C}$ & $12.08^{\circ} \mathrm{C}$ & $12.23^{\circ} \mathrm{C}$ & $12.14^{\circ} \mathrm{C}$ \\
\hline
\end{tabular}

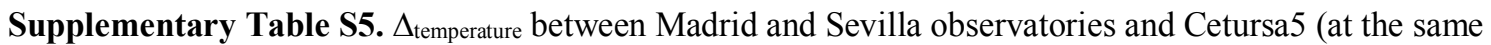
elevation as LdRS, 3020 masl) calculated using different approaches: 1) using equation from Fig. S1b from the mean values between $\Delta_{\text {MMAT }}$ and $\Delta_{\text {elev }}$ among all the studied low elevation observatories $v s$ those from high elevation areas; 2) the same as the previous one but with the $\Delta_{\text {MAAT }}$ (equation from Fig. S1d); 3) using equations from Fig. S1f and j (Sevilla-Madrid respectively) from the mean values between $\Delta_{\text {MMAT }}$ and $\Delta_{\text {elev }}$ of Sevilla or Madrid observatories respectively $v s$ those from high elevation areas; 4) the same as the

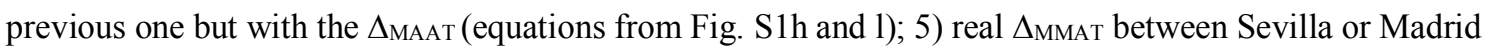
observatories and Cetursa 5 observatory $(3020$ masl $)$; 6 ) the same as the previous one but with the $\Delta$ MAAT. 


\begin{tabular}{|c|c|c|c|c|}
\hline $\begin{array}{c}\text { LdRS she } \\
n=32\end{array}$ & $\mathrm{C}_{28}$ 1,13-diol & $C_{30}$ 1,15-diol & $\mathrm{C}_{30}$ 1,13-diol & $\mathrm{C}_{32}$ 1,15-diol \\
\hline$C_{28}$ 1,13-diol & & $4.8 \mathrm{E}-06$ & $1.8 \mathrm{E}-07$ & $9.4 \mathrm{E}-14$ \\
\hline $\mathrm{C}_{30}$ 1,15-diol & -0.71 & & 0.005 & 0.001 \\
\hline $\mathrm{C}_{30}$ 1,13-diol & 0.78 & -0.49 & & $1.2 \mathrm{E}-17$ \\
\hline $\mathrm{C}_{32}$ 1,15-diol & -0.92 & 0.57 & -0.96 & \\
\hline $\begin{array}{c}\text { LdRS lge } \\
n=20\end{array}$ & $\mathrm{C}_{28}$ 1,13-diol & $\mathrm{C}_{30}$ 1,15-diol & $\mathrm{C}_{30}$ 1,13-diol & $C_{32}$ 1,15-diol \\
\hline $\mathrm{C}_{28}$ 1,13-diol & & 0.02 & $1.2 \mathrm{E}-09$ & $9.2 \mathrm{E}-14$ \\
\hline $\mathrm{C}_{30}$ 1,15-diol & -0.53 & & 0.07 & 0.07 \\
\hline $\mathrm{C}_{30}$ 1,13-diol & 0.94 & -0.42 & & $5.5 \mathrm{E}-14$ \\
\hline $\mathrm{C}_{32}$ 1,15-diol & -0.98 & 0.42 & -0.98 & \\
\hline
\end{tabular}

Supplementary Table S6. Pearson correlation table $(r v s p)$ for the relative abundances of the dominant LCDs in both short and long LdRS cores (she and lgc). 


\begin{tabular}{|c|c|c|c|c|}
\hline $\begin{array}{c}\text { LCDs vs } \\
\text { reference } \\
\text { MAAT at 3020 masl }\end{array}$ & \multicolumn{2}{|c|}{ LdRS she } & \multicolumn{2}{c|}{$\begin{array}{c}\text { LdRS } \\
\text { shc+lgc }\end{array}$} \\
\cline { 2 - 5 } & $r$ & $p$ & $r$ & $p$ \\
\hline LDI & 0.90 & $2 \mathrm{E}-28$ & 0.90 & $2 \mathrm{E}-36$ \\
\hline $\mathbf{C}_{\mathbf{2 8}} \mathbf{1 , 1 3 - d i o l}$ & -0.85 & $2 \mathrm{E}-22$ & -0.72 & $1 \mathrm{E}-17$ \\
\hline $\mathbf{C}_{\mathbf{3 0}} \mathbf{1 , 1 5 - d i o l}$ & 0.37 & $9 \mathrm{E}-04$ & 0.32 & $9 \mathrm{E}-04$ \\
\hline $\mathbf{C}_{\mathbf{3 0}} \mathbf{1 , 1 3 - d i o l}$ & -0.90 & $4 \mathrm{E}-29$ & -0.86 & $4 \mathrm{E}-32$ \\
\hline $\mathbf{C}_{\mathbf{3 2}} \mathbf{1 , 1 5}-$ diol & 0.89 & $2 \mathrm{E}-27$ & 0.79 & $2 \mathrm{E}-23$ \\
\hline
\end{tabular}

Supplementary Table S7. Pearson correlations between the relative abundances of the dominant LCDs in both short and long LdRS cores (shc and lgc), the LDI values, and the mean reconstructed reference temperature time-series at 3020 masl for the last $\sim 100$ years. 


\begin{tabular}{|c|c|c|c|c|}
\hline Calibrations & Variable & $r$ & $p$ & $r^{2}$ \\
\hline \multirow{4}{*}{$\begin{array}{l}\text { Calibration 1: } \\
\text { Fractional abundances of LCDs: }\end{array}$} & $\mathrm{C}_{28}$ 1,13-diol & -0.72 & $1 \mathrm{E}-17$ & 0.51 \\
\hline & $\mathrm{C}_{30}$ 1,15-diol & 0.32 & 9E-04 & 0.10 \\
\hline & $\mathrm{C}_{30}$ 1,13-diol & -0.86 & $4 \mathrm{E}-32$ & 0.75 \\
\hline & $\mathrm{C}_{32}$ 1,15-diol & 0.79 & $2 \mathrm{E}-23$ & 0.62 \\
\hline \multirow{4}{*}{$\begin{array}{l}\text { Calibration } 2 \text { and 3: } \\
\text { Ratio of fractional abundances of LCDs: }\end{array}$} & $\mathrm{C}_{30} 1,15-/ \mathrm{C}_{28} 1,13$-diols & 0.87 & $8 \mathrm{E}-34$ & 0.76 \\
\hline & $\mathrm{C}_{30} 1,15-$ / $\mathrm{C}_{30} 1,13$-diols & 0.89 & $6 \mathrm{E}-37$ & 0.80 \\
\hline & $\mathrm{C}_{32}$ 1,15- / $\mathrm{C}_{28}$ 1,13-diols & 0.85 & $1 \mathrm{E}-29$ & 0.72 \\
\hline & $\mathrm{C}_{32} 1,15-/ \mathrm{C}_{30} 1,13$-diols & 0.87 & $3 E-33$ & 0.76 \\
\hline
\end{tabular}

Supplementary Table S8. Comparison between the different statistics of the potential LCD-based temperature calibrations computed by means of multiple linear regressions and the reference temperature time-series at 3020 masl for the last $\sim 100$ years. The obtained calibration equations are:

- MLR Calibration 1:

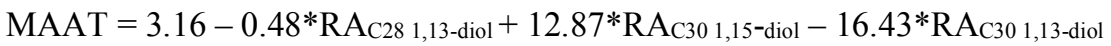
$r^{2}=0.78$

- $\quad$ MLR Calibration 2:

MAAT $=0.005-1.14 * \mathrm{RA}_{(\mathrm{C} 301,15-/ \mathrm{C} 281,13 \text {-diols })}+4.21 * \mathrm{RA}_{\left(\mathrm{C} 301,15-/ \mathrm{C}_{0} 01,13 \text {-diols }\right)}+0.14 * \mathrm{RA}_{(\mathrm{C} 321,15-/}$ C28 1,13-diols) $-0.12 * \mathrm{RA}_{(\mathrm{C} 32} 1,15$-/ C30 1,13-diols) $r^{2}=0.83$

- MLR Calibration 3:

MAAT $=0.03+0.74 * \mathrm{RA}_{\left(\mathrm{C} 301,15-/ \mathrm{C}_{28} 1,13 \text {-diols }\right)}+2.44 * \mathrm{RA}_{(\mathrm{C} 30}$ 1,15-/ C30 1,13-diols $)$ $r^{2}=0.81$ 


\begin{tabular}{|c|c|c|c|c|c|c|c|c|}
\hline LdRS $v s$ & Lake sed & $\begin{array}{c}\text { Marine } \\
\text { sed }\end{array}$ & $\begin{array}{c}\text { River } \\
\text { sed }\end{array}$ & $\begin{array}{c}\text { River } \\
\text { SPM }\end{array}$ & $\begin{array}{c}\text { Marine } \\
\text { algae }\end{array}$ & Soil algae & Snow algae & Fresh-w algae \\
\hline $\begin{array}{c}\mathrm{C}_{28} 1,13- \\
\text { diol }\end{array}$ & $\mathrm{ns}$ & $\mathrm{ns}$ & $* * *$ & $\mathrm{~ns}$ & $* * *$ & $* *$ & $\mathrm{~ns}$ & $* * *$ \\
\hline $\begin{array}{c}\mathrm{C}_{30} 1,13- \\
\text { diol }\end{array}$ & $* * *$ & $* *$ & $*$ & $* * *$ & $\mathrm{~ns}$ & $* *$ & $* *$ & $* *$ \\
\hline $\begin{array}{c}\mathrm{C}_{30} 1,15- \\
\text { diol }\end{array}$ & $* * *$ & $* * *$ & $* * *$ & $* * *$ & $\mathrm{~ns}$ & $* * *$ & $* *$ & $* *$ \\
\hline $\begin{array}{c}\mathrm{C}_{32} 1,15- \\
\text { diol }\end{array}$ & $* * *$ & $* * *$ & $* * *$ & $* * *$ & $*$ & $* *$ & $\S$ & $\mathrm{ns}$ \\
\hline
\end{tabular}

Supplementary Table S9. Results of the Mann-Whitney U test that has been performed to identify the differences between individual isomers from LdRS and those from other sources. Literature data from: lake sediments (Rampen et al., 2014a), algal cultures (Rampen et al., 2014a), marine sediments (de Bar et al., 2016; Lattaud et al., 2017a; Rampen et al., 2014b; Rampen et al., 2012), river sediments/inputs (de Bar et al., 2016; Lattaud et al., 2017b), river particulate organic matter (Lattaud et al., 2018). Significant level for the differences between LdRS and other source groups: ${ }^{* * *} p<0.001 ;{ }^{* *} 0.001<p<0.01 ; * 0.01<p<$ $.05 ;{ }^{\S} 0.05<p<0.1 ;{ }^{\text {ns }} p>0.1$. 


\begin{tabular}{|c|c|c|c|c|c|c|c|c|c|}
\hline \multirow{2}{*}{\multicolumn{2}{|c|}{ LdRS she LDI vs }} & \multirow{3}{*}{$\begin{array}{c}\mathbf{n} \\
32\end{array}$} & \multirow{2}{*}{\multicolumn{2}{|c|}{$\begin{array}{c}\text { Normal } \\
\text { correlation }\end{array}$}} & \multicolumn{2}{|c|}{$\begin{array}{c}{\text { (z-score })^{2}} \\
\text { correaltion }\end{array}$} & \multirow{2}{*}{$\begin{array}{c}\begin{array}{c}\text { Mann- } \\
\text { Kendall }\end{array} \\
\begin{array}{c}\text { no trend } \\
\boldsymbol{p}>\mathbf{0 . 0 5}\end{array} \\
0.96\end{array}$} & \multicolumn{2}{|c|}{$\begin{array}{l}\text { Detrended } \\
\text { correlation }\end{array}$} \\
\hline & & & & & & & & & \\
\hline Solar & $\Delta$ TSI & & $\frac{r}{0.58}$ & \begin{tabular}{|c}
$\boldsymbol{p}$ \\
0.0004
\end{tabular} & $\begin{array}{c}\boldsymbol{r} \\
-0.10\end{array}$ & $\begin{array}{c}\boldsymbol{p} \\
0.5891\end{array}$ & & $\frac{r}{-0.03}$ & $\frac{\boldsymbol{p}}{0.8893}$ \\
\hline & TSI & 32 & 0.56 & 0.0009 & 0.10 & 0.5675 & 0.87 & 0.02 & 0.9120 \\
\hline \multirow[t]{3}{*}{ Volcanic } & NH volcanic aerosol & 27 & 0.15 & 0.4201 & 0.09 & 0.6435 & 0.66 & 0.03 & 0.8677 \\
\hline & Global volcanic aerosol & 27 & 0.19 & 0.3235 & -0.002 & 0.9926 & 0.93 & 0.06 & 0.7584 \\
\hline & *Global volcanic forcing & 27 & -0.04 & 0.8442 & 0.04 & 0.8392 & 0.59 & 0.12 & 0.5181 \\
\hline \multirow[t]{2}{*}{ Atmospheric } & NAO & 30 & -0.03 & 0.8830 & 0.33 & 0.0772 & $3 \mathrm{E}-05$ & 0.05 & 0.7760 \\
\hline & AMO & 32 & 0.61 & 0.0002 & 0.41 & 0.0210 & 0.37 & 0.32 & 0.0711 \\
\hline \multirow{3}{*}{$\begin{array}{l}\text { Green house } \\
\text { gases }\end{array}$} & $\mathrm{CO}_{2}(\mathrm{ppm})$ & 31 & 0.83 & $6 \mathrm{E}-09$ & 0.77 & 5E-07 & 0.36 & 0.70 & 0.0000 \\
\hline & $\mathrm{NO}_{2}(\mathrm{ppm})$ & 31 & 0.85 & 1E-09 & 0.78 & $2 \mathrm{E}-07$ & 0.46 & 0.71 & 0.0000 \\
\hline & $\mathrm{CH}_{4}(\mathrm{ppm})$ & 31 & 0.86 & $5 \mathrm{E}-10$ & 0.73 & $2 \mathrm{E}-06$ & 0.56 & 0.65 & 0.0001 \\
\hline \multirow[t]{3}{*}{ Temperatures } & CPS Summer temperatures & 32 & 0.58 & 0.0007 & 0.44 & 0.0131 & 0.30 & 0.40 & 0.0261 \\
\hline & $\mathbf{U}^{\mathrm{K}^{\prime}{ }_{37}-\mathrm{SST} \text { Gol-Ho1B }}$ & 32 & 0.76 & 4E-07 & 0.24 & 0.1884 & 0.83 & 0.16 & 0.3739 \\
\hline & Global Temperatures (GLSS) & 19 & 0.89 & $3 \mathrm{E}-07$ & 0.69 & 0.0010 & 0.62 & 0.74 & 0.0003 \\
\hline
\end{tabular}

Supplementary Table S10. Pearson correlations (normal and detrended) between the LDI record from the LdRS short core and different proxies for solar and volcanic forcing, North Atlantic modes, greenhouse gases, and temperatures. Normal correlations show long-term trends. Data were standardised (z-scores), normalised (squares) and a Mann-Kendall trend test was performed using PAST software (Hammer et al., 2001) in order to assess the existence of any trend over time in the data series. Afterwards, data were detrended by fitting a linear regression versus time, and a Pearson correlation was worked out with the residuals. *: Note that inverse global volcanic forcing values have been used in order to show the same trends as in Fig. 5.

Solar Proxies: $\Delta$ TSI, reconstruction of the difference of the total solar irradiance from the value of the PMOD composite series during the solar cycle minimum of the year $1986 \mathrm{CE}\left(1365.57 \mathrm{~W} \mathrm{~m}^{-2}\right.$ ) (Steinhilber et al., 2009); TSI, total solar Irradiance (Coddington et al., 2016).

Volcanic proxies: Annual stratospheric volcanic sulfate aerosol injection for the past 1500 years in the North Hemisphere and worldwide (Gao et al., 2008); global volcanic aerosol forcing (W $\mathrm{m}^{-2}$ ) (Sigl et al., 2015).

North Atlantic modes: NAO, North Atlantic Oscillation reconstruction (Trouet et al., 2009); AMO, Atlantic Multidecadal Oscillation reconstruction (Mann et al., 2009).

Greenhouse gases: Reconstructed concentrations of atmospheric $\mathrm{CO}_{2}, \mathrm{NO}_{2}$, and $\mathrm{CH}_{4}$ (ppm) (Schmidt et al., 2011).

Temperatures: Composite-plus-scaling (CPS) mean summer temperature anomaly reconstruction from tree rings records in Europe with respect to 1974-2003 CE (MSTA ${ }^{\circ} \mathrm{C}$ ) (Luterbacher et al., 2016); $\mathrm{U}^{\mathrm{K}}{ }^{\prime}{ }_{37-}$ derived SSTs from the marine record in the Gulf of Lion (NW Mediterranean Sea) (Sicre et al., 2016), and global land and sea surface (GLSS) mean annual temperature anomalies with respect to 1979-2008 CE (Hansen et al., 2010). 


\begin{tabular}{|c|c|c|c|c|c|c|c|c|c|}
\hline \multirow{2}{*}{\multicolumn{2}{|c|}{ LdRS lgc LDI vs }} & \multirow{3}{*}{$\begin{array}{c}\text { n } \\
16\end{array}$} & \multicolumn{2}{|c|}{$\begin{array}{c}\text { Normal } \\
\text { correlation }\end{array}$} & \multicolumn{2}{|c|}{$\begin{array}{c}{\text { (z-score })^{2}} \\
\text { correaltion }\end{array}$} & \multirow{3}{*}{$\begin{array}{c}\begin{array}{c}\text { Mann- } \\
\text { Kendall }\end{array} \\
\begin{array}{c}\text { no trend } \\
\boldsymbol{p}>\mathbf{0 . 0 5}\end{array} \\
0.19\end{array}$} & \multicolumn{2}{|c|}{$\begin{array}{l}\text { Detrended } \\
\text { correlation }\end{array}$} \\
\hline & & & \multirow{2}{*}{$\frac{r}{-0.73}$} & \multirow{2}{*}{\begin{tabular}{|c|}
$\boldsymbol{p}$ \\
0.0015
\end{tabular}} & \multirow{2}{*}{$\frac{r}{r}$} & \multirow{2}{*}{$\begin{array}{c}\boldsymbol{p} \\
0.1301\end{array}$} & & \multirow{2}{*}{$\frac{\boldsymbol{r}}{0.38}$} & \multirow{2}{*}{$\frac{p}{0.1454}$} \\
\hline Solar & $* \Delta^{14} \mathrm{C}$ & & & & & & & & \\
\hline & $\Delta$ TSI & 20 & 0.69 & 0.0007 & 0.38 & 0.1024 & 0.02 & 0.367 & 0.1117 \\
\hline & TSI & 13 & 0.88 & 0.0001 & 0.13 & 0.0863 & 0.85 & 0.56 & 0.0451 \\
\hline \multirow[t]{3}{*}{ Volcanic } & NH volcanic aerosol & 19 & -0.07 & 0.7758 & 0.13 & 0.5932 & 0.16 & 0.13 & 0.5932 \\
\hline & Global volcanic aerosol & 19 & 0.06 & 0.7922 & 0.28 & 0.2517 & 0.05 & 0.28 & 0.2517 \\
\hline & *Global volcanic forcing & 16 & -0.03 & 0.8948 & 0.32 & 0.1564 & 0.07 & 0.33 & 0.1564 \\
\hline \multirow[t]{2}{*}{ North Atlantic modes } & NAO & 16 & 0.10 & 0.7127 & 0.33 & 0.2098 & 0.30 & 0.23 & 0.3890 \\
\hline & AMO & 19 & 0.60 & 0.0064 & 0.37 & 0.1156 & 0.48 & 0.25 & 0.2928 \\
\hline \multirow[t]{3}{*}{ Greenhouse gases } & $\mathrm{CO}_{2}(\mathrm{ppm})$ & 20 & 0.74 & 0.0002 & 0.73 & 0.0003 & 0.97 & 0.65 & 0.0018 \\
\hline & $\mathrm{NO}_{2}(\mathrm{ppm})$ & 20 & 0.65 & 0.0018 & 0.68 & 0.0009 & 0.92 & 0.61 & 0.0043 \\
\hline & $\mathrm{CH}_{4}(\mathrm{ppm})$ & 20 & 0.73 & 0.0003 & 0.77 & 0.0001 & 0.35 & 0.71 & 0.0005 \\
\hline \multirow[t]{6}{*}{ Temperatures } & CPS Summer temperatures & 20 & 0.71 & 0.0005 & 0.46 & 0.0420 & 0.18 & 0.34 & 0.1421 \\
\hline & $\mathrm{U}^{\mathrm{K}}{ }_{37-}{ }^{-S S T}$ Gol-Ho1 B & 20 & 0.61 & 0.0043 & 0.72 & 0.0003 & 0.12 & 0.65 & 0.0018 \\
\hline & $\mathrm{U}^{\mathrm{K}^{\prime}{ }_{37-S S T} \text { TTR-17-384B }}$ & 17 & 0.24 & 0.3581 & 0.02 & 0.9374 & 0.77 & 0.01 & 0.9537 \\
\hline & 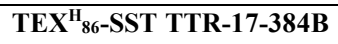 & 17 & 0.45 & 0.0707 & 0.53 & 0.0299 & 0.97 & 0.47 & 0.0538 \\
\hline & $\mathrm{U}^{\mathrm{K}^{\prime}{ }_{37-S S T} \mathrm{TTR}-17-436 \mathrm{~B}}$ & 18 & 0.34 & 0.1697 & 0.68 & 0.0020 & 0.32 & 0.70 & 0.0013 \\
\hline & TEX $^{\mathrm{H}_{86}-S S T ~ T T R-17-436 B ~}$ & 18 & 0.43 & 0.0721 & 0.18 & 0.4830 & 0.40 & 0.34 & 0.1610 \\
\hline
\end{tabular}

Supplementary Table S11. Pearson correlations (normal and detrended) between the LDI record from the LdRS long core and different proxies for solar and volcanic forcing, North Atlantic modes, greenhouse gases, and temperatures. Normal correlations show long-term trends. Data were standardised (z-scores), normalised (squares), and a Mann-Kendall trend test was performed using PAST software (Hammer et al., 2001) in order to assess the existence of any trend over time in the data series. Afterwards, data were detrended by fitting a linear regression versus time, and a Pearson correlation was worked out with the residuals. *: Note that inverse $\Delta^{14} \mathrm{C}$ and global volcanic forcing values have been used in order to show the same trends as in Fig. 6.

Solar Proxies: $\Delta^{14} \mathbf{C}$ (Reimer et al., 2013); $\Delta$ TSI, reconstruction of the difference of the total solar irradiance from the value of the PMOD composite series during the solar cycle minimum of the year 1986 CE (1365.57 W m ${ }^{-2}$ ) (Steinhilber et al., 2009); TSI, total solar irradiance (Coddington et al., 2016).

Volcanic proxies: Annual stratospheric volcanic sulfate aerosol injection for the past 1500 years in the North Hemisphere, and worldwide (Gao et al., 2008); global volcanic aerosol forcing ( $\mathrm{W} \mathrm{m}^{-2}$ ) (Sigl et al., 2015).

North Atlantic modes: NAO, North Atlantic Oscillation reconstruction (Trouet et al., 2009); AMO, Atlantic Multidecadal Oscillation reconstruction (Mann et al., 2009).

Greenhouse gases: Reconstructed concentrations of atmospheric $\mathrm{CO}_{2}, \mathrm{NO}_{2}$, and $\mathrm{CH}_{4}(\mathrm{ppm})$ (Schmidt et al., 2011).

Temperatures: Composite-plus-scaling (CPS) mean summer temperature anomaly reconstruction from tree rings records in Europe with respect to $1974-2003 \mathrm{CE}$ (MSTA ${ }^{\circ} \mathrm{C}$ ); $\mathrm{U}^{\mathrm{K}}{ }_{37}$-derived SSTs from the marine record in the Gulf of Lion (NW Mediterranean Sea) (Sicre et al., 2016); $\mathrm{U}^{\mathrm{K}}{ }_{37}$-and TEX ${ }_{86}$-derived SSTs records from cores 384B and 436B in the Alboran Sea (Nieto-Moreno et al., 2013). 


\begin{tabular}{|c|c|c|c|c|c|c|c|c|}
\hline & \multirow{2}{*}{ LdRS she vs } & \multirow{2}{*}{$\mathbf{n}$} & \multicolumn{2}{|c|}{$\begin{array}{c}\text { MLR } \\
\text { calibration 1 }\end{array}$} & \multicolumn{2}{|c|}{$\begin{array}{c}\text { MLR } \\
\text { calibration } 2\end{array}$} & \multicolumn{2}{|c|}{$\begin{array}{c}\text { MLR } \\
\text { calibration } 3\end{array}$} \\
\hline & & & $r$ & $p$ & $r$ & $p$ & $r$ & $p$ \\
\hline \multirow{2}{*}{ Solar } & $\Delta \mathrm{TSI}$ & 32 & 0.69 & $1.3 \mathrm{E}-05$ & 0.60 & $2.6 \mathrm{E}-04$ & 0.59 & 4.1E-04 \\
\hline & TSI & 32 & 0.63 & $1.1 \mathrm{E}-04$ & 0.55 & $1.1 \mathrm{E}-03$ & 0.55 & $1.0 \mathrm{E}-03$ \\
\hline \multirow{3}{*}{ Volcanic } & NH volcanic aerosol & 27 & 0.13 & 0.4912 & 0.13 & 0.4843 & 0.14 & 0.4523 \\
\hline & Global volcanic aerosol & 27 & 0.15 & 0.4232 & 0.17 & 0.3581 & 0.18 & 0.3438 \\
\hline & *Global volcanic forcing & 27 & 0.04 & 0.8212 & -0.01 & 0.9597 & -0.02 & 0.9168 \\
\hline \multirow{2}{*}{ Atmospheric } & NAO & 30 & 0.01 & 0.9784 & 0.08 & 0.6620 & 0.03 & 0.8939 \\
\hline & AMO & 32 & 0.71 & 4.3E-06 & 0.69 & $1.2 \mathrm{E}-05$ & 0.63 & $9.8 \mathrm{E}-05$ \\
\hline \multirow{3}{*}{$\begin{array}{c}\text { Green house } \\
\text { gases }\end{array}$} & $\mathrm{CO}_{2}(\mathrm{ppm})$ & 31 & 0.92 & $2.5 \mathrm{E}-13$ & 0.90 & $2.8 \mathrm{E}-12$ & 0.86 & $4.6 \mathrm{E}-10$ \\
\hline & $\mathrm{NO}_{2}(\mathbf{p p m})$ & 31 & 0.93 & $2.7 \mathrm{E}-14$ & 0.92 & $2.6 \mathrm{E}-13$ & 0.88 & $6.6 \mathrm{E}-11$ \\
\hline & $\mathrm{CH}_{4}(\mathrm{ppm})$ & 31 & 0.94 & $4.9 \mathrm{E}-15$ & 0.92 & 4.7E-13 & 0.88 & $6.3 \mathrm{E}-11$ \\
\hline \multirow{3}{*}{ Temperatures } & CPS Summer temperatures & 32 & 0.66 & $5.2 \mathrm{E}-05$ & 0.65 & $8.9 \mathrm{E}-05$ & 0.61 & $2.9 \mathrm{E}-04$ \\
\hline & $\mathrm{U}^{\mathrm{K}^{\prime}{ }_{37}-\mathrm{SST} \text { Gol-Ho1B }}$ & 32 & 0.86 & $2.2 \mathrm{E}-10$ & 0.80 & 2.9E-08 & 0.77 & $2.4 \mathrm{E}-07$ \\
\hline & Global Temperatures (GLSS) & 19 & 0.90 & 7.4E-09 & 0.93 & $1.0 \mathrm{E}-10$ & 0.90 & 4.2E-09 \\
\hline
\end{tabular}

Supplementary Table S12. Pearson correlations between the reconstructed tempertures in the LdRS shc from MLR calibrations 1, 2, and 3 and different proxies for solar and volcanic forcing, North Atlantic modes, greenhouse gases, and temperatures. Note that inverse global volcanic forcing values have been used in order to show the same trends as in Fig. 5. Proxy explanation as in Supplementary Table S10. 


\begin{tabular}{|c|c|c|c|c|c|c|c|c|}
\hline \multirow{2}{*}{\multicolumn{2}{|c|}{ LdRS lgc $v s$}} & \multirow{2}{*}{$\mathbf{n}$} & \multicolumn{2}{|c|}{$\begin{array}{c}\text { MLR } \\
\text { calibration 1 }\end{array}$} & \multicolumn{2}{|c|}{$\begin{array}{c}\text { MLR } \\
\text { calibration } 2 \\
\end{array}$} & \multicolumn{2}{|c|}{$\begin{array}{c}\text { MLR } \\
\text { calibration } 3 \\
\end{array}$} \\
\hline & & & $r$ & $p$ & $r$ & $p$ & $r$ & $p$ \\
\hline \multirow{3}{*}{ Solar } & $* \Delta^{14} \mathrm{C}$ & 16 & -0.57 & 0.0202 & -0.73 & 0.0013 & -0.73 & 0.0012 \\
\hline & $\Delta \mathbf{T S I}$ & 20 & 0.71 & 0.0005 & 0.71 & 0.0004 & 0.71 & 0.0005 \\
\hline & TSI & 13 & 0.84 & 0.0003 & 0.86 & 0.0001 & 0.86 & 0.0002 \\
\hline \multirow{3}{*}{ Volcanic } & NH volcanic aerosol & 19 & 0.09 & 0.7058 & 0.06 & 0.7959 & 0.01 & 0.9723 \\
\hline & Global volcanic aerosol & 19 & 0.05 & 0.8245 & 0.16 & 0.5235 & 0.13 & 0.5925 \\
\hline & *Global volcanic forcing & 16 & 0.05 & 0.8287 & -0.08 & 0.7238 & -0.08 & 0.7486 \\
\hline \multirow{2}{*}{ North Atlantic modes } & NAO & 16 & -0.01 & 0.9746 & 0.15 & 0.5775 & 0.13 & 0.6378 \\
\hline & AMO & 19 & 0.52 & 0.0225 & 0.63 & 0.0036 & 0.61 & 0.0054 \\
\hline \multirow{3}{*}{ Greenhouse gases } & $\mathrm{CO}_{2}(\mathrm{ppm})$ & 20 & 0.74 & 0.0002 & 0.76 & 0.0001 & 0.77 & 0.0001 \\
\hline & $\mathrm{NO}_{2}(\mathrm{ppm})$ & 20 & 0.74 & 0.0002 & 0.68 & 0.0009 & 0.69 & 0.0009 \\
\hline & $\mathrm{CH}_{4}(\mathrm{ppm})$ & 20 & 0.73 & 0.0003 & 0.75 & 0.0002 & 0.76 & 0.0001 \\
\hline \multirow{6}{*}{ Temperatures } & CPS Summer temperatures & 20 & 0.72 & 0.0004 & 0.75 & 0.0001 & 0.73 & 0.0002 \\
\hline & $\mathrm{U}^{\mathrm{K}}{ }_{37-\mathrm{SST}}$ Gol-Ho1B & 20 & 0.63 & 0.0031 & 0.65 & 0.0020 & 0.65 & 0.0019 \\
\hline & $\mathrm{U}^{\mathrm{K}}{ }_{37}{ }^{-S S T} \mathrm{TTR}-17-384 \mathrm{~B}$ & 17 & 0.38 & 0.1342 & 0.20 & 0.4464 & 0.19 & 0.4703 \\
\hline & TEX $^{H_{86}-S S T ~ T T R-17-384 B ~}$ & 17 & 0.20 & 0.4314 & 0.46 & 0.0605 & 0.47 & 0.0552 \\
\hline & $\mathrm{U}^{\mathrm{K}}{ }_{37-\mathrm{SST}} \mathrm{TTR}-17-436 \mathrm{~B}$ & 18 & 0.39 & 0.1103 & 0.41 & 0.0951 & 0.37 & 0.1274 \\
\hline & TEX $^{H_{86}-S S T}$ TTR-17-436B & 18 & 0.30 & 0.2318 & 0.51 & 0.0305 & 0.47 & 0.0516 \\
\hline
\end{tabular}

Supplementary Table S13. Pearson correlations between recontructed tempertures in the LdRS lgc from calibrations 1, 2, and 3 and different proxies for solar and volcanic forcing, North Atlantic modes, greenhouse gases, and temperatures. Note that inverse $\Delta^{14} \mathrm{C}$ and global volcanic forcing values have been used in order to show the same trends as in Fig. 6. Proxy explanation as in Supplementary Table S11. 


\section{Supplementary References}

Coddington, O., Lean, J. L., Pilewskie, P., Snow, M., and Lindholm, D.: A Solar Irradiance Climate Data Record, Bulletin of the American Meteorological Society, 97, 1265-1282, 2016.

de Bar, M. W., Dorhout, D. J. C., Hopmans, E. C., Rampen, S. W., Sinninghe Damsté, J. S., and Schouten, S.: Constraints on the application of long chain diol proxies in the Iberian Atlantic margin, Organic Geochemistry, 101, 184-195, 2016.

Dogniaux, R.: Prediction of Solar Radiation in Areas with a Specific Microclimate, Prediction of Solar Radiation in Areas with a Specific Microclimate, 1994.

Gao, C., Robock, A., and Ammann, C.: Volcanic forcing of climate over the past 1500 years: An improved ice core-based index for climate models, Journal of Geophysical Research: Atmospheres, 113, 2008.

Gonzalez-Hidalgo, J. C., Peña-Angulo, D., Brunetti, M., and Cortesi, N.: MOTEDAS: a new monthly temperature database for mainland Spain and the trend in temperature (1951-2010), International Journal of Climatology, 35, 4444-4463, 2015.

Hammer, Ø., Harper, D. A. T., and Ryan, P. D.: PAST: Paleontological statistics software package for education and data analysis, Palaeontologia Electronica 4, 9, 2001.

Hansen, J., Ruedy, R., Sato, M., and Lo, K.: Global Surface Temperature Change, Reviews of Geophysics, 48, 2010.

International-Civil-Aviation-Organization: Manual of the ICAO standard atmosphere : extended to 80 kilometres (262 500 feet) (Third ed.), International Civil Aviation Organization, Montreal, Quebec, 1993.

Lattaud, J., Dorhout, D., Schulz, H., Castañeda, I. S., Schefuß, E., Sinninghe Damsté, J. S., and Schouten, S.: The C32 alkane-1,15-diol as a proxy of late Quaternary riverine input in coastal margins, Clim. Past, 13, 1049-1061, 2017a.

Lattaud, J., Kim, J.-H., De Jonge, C., Zell, C., Sinninghe Damsté, J. S., and Schouten, S.: The C32 alkane-1,15-diol as a tracer for riverine input in coastal seas, Geochimica et Cosmochimica Acta, 202, 146-158, 2017b.

Lattaud, J., Kirkels, F., Peterse, F., Freymond, C. V., Eglinton, T. I., Hefter, J., Mollenhauer, G., Balzano, S., Villanueva, L., van der Meer, M. T. J., Hopmans, E. C., Sinninghe Damsté, J. S., and Schouten, S.: Long-chain diols in rivers: distribution and potential biological sources, Biogeosciences, 15, 4147-4161, 2018.

Luterbacher, J., Werner, J. P., Smerdon, J. E., Fernández-Donado, L., González-Rouco, F. J., Barriopedro, D., Ljungqvist, F. C., Büntgen, U., Zorita, E., Wagner, S., Esper, J., McCarroll, D., Toreti, A., Frank, D., Jungclaus, J. H., Barriendos, M., Bertolin, C., Bothe, O., Brázdil, R., Camuffo, D., Dobrovolný, P., Gagen, M., GarcíaBustamante, E., Ge, Q., Gómez-Navarro, J. J., Guiot, J., Hao, Z., Hegerl, G. C., Holmgren, K., Klimenko, V. V., Martín-Chivelet, J., Pfister, C., Roberts, N., Schindler, A., Schurer, A., Solomina, O., von Gunten, L., Wahl, E., Wanner, H., Wetter, O., Xoplaki, E., Yuan, N., Zanchettin, D., Zhang, H., and Zerefos, C.: European summer temperatures since Roman times, Environmental Research Letters, 11, 024001, 2016. 
Mann, M. E., Zhang, Z., Rutherford, S., Bradley, R. S., Hughes, M. K., Shindell, D., Ammann, C., Faluvegi, G., and Ni, F.: Global Signatures and Dynamical Origins of the Little Ice Age and Medieval Climate Anomaly, Science, 326, 1256-1260, 2009.

Nieto-Moreno, V., Martínez-Ruiz, F., Willmott, V., García-Orellana, J., Masqué, P., and Sinninghe Damsté, J. S.: Climate conditions in the westernmost Mediterranean over the last two millennia: An integrated biomarker approach, Organic Geochemistry, $55,1-10,2013$.

Observatorio del cambio global de Sierra Nevada: Linaria v1.0. iEcolab - Laboratorio de Ecología Terrestre - Universidad de Granada, http://linaria.obsnev.es, 2019.

Rampen, S. W., Datema, M., Rodrigo-Gámiz, M., Schouten, S., Reichart, G.-J., and Sinninghe Damsté, J. S.: Sources and proxy potential of long chain alkyl diols in lacustrine environments, Geochimica et Cosmochimica Acta, 144, 59-71, 2014a.

Rampen, S. W., Willmott, V., Kim, J.-H., Rodrigo-Gámiz, M., Uliana, E., Mollenhauer, G., Schefuß, E., Sinninghe Damsté, J. S., and Schouten, S.: Evaluation of long chain 1,14-alkyl diols in marine sediments as indicators for upwelling and temperature, Organic Geochemistry, 76, 39-47, 2014 b.

Rampen, S. W., Willmott, V., Kim, J.-H., Uliana, E., Mollenhauer, G., Schefuß, E., Sinninghe Damsté, J. S., and Schouten, S.: Long chain 1,13- and 1,15-diols as a potential proxy for palaeotemperature reconstruction, Geochimica et Cosmochimica Acta, 84, 204-216, 2012.

Reimer, P. J., Bard, E., Bayliss, A., Beck, J. W., Blackwell, P. G., Ramsey, C. B., Buck, C. E., Cheng, H., Edwards, R. L., Friedrich, M., Grootes, P. M., Guilderson, T. P., Haflidason, H., Hajdas, I., Hatté, C., Heaton, T. J., Hoffmann, D. L., Hogg, A. G., Hughen, K. A., Kaiser, K. F., Kromer, B., Manning, S. W., Niu, M., Reimer, R. W., Richards, D. A., Scott, E. M., Southon, J. R., Staff, R. A., Turney, C. S. M., and van der Plicht, J.: IntCal13 and Marine13 Radiocarbon Age Calibration Curves 050,000 Years cal BP, Radiocarbon, 55, 1869-1887, 2013.

Schmidt, G. A., Jungclaus, J. H., Ammann, C. M., Bard, E., Braconnot, P., Crowley, T. J., Delaygue, G., Joos, F., Krivova, N. A., Muscheler, R., Otto-Bliesner, B. L., Pongratz, J., Shindell, D. T., Solanki, S. K., Steinhilber, F., and Vieira, L. E. A.: Climate forcing reconstructions for use in PMIP simulations of the last millennium (v1.0), Geosci. Model Dev., 4, 33-45, 2011.

Sicre, M.-A., Jalali, B., Martrat, B., Schmidt, S., Bassetti, M.-A., and Kallel, N.: Sea surface temperature variability in the North Western Mediterranean Sea (Gulf of Lion) during the Common Era, Earth and Planetary Science Letters, 456, 124-133, 2016.

Sigl, M., Winstrup, M., McConnell, J. R., Welten, K. C., Plunkett, G., Ludlow, F., Buntgen, U., Caffee, M., Chellman, N., Dahl-Jensen, D., Fischer, H., Kipfstuhl, S., Kostick, C., Maselli, O. J., Mekhaldi, F., Mulvaney, R., Muscheler, R., Pasteris, D. R., Pilcher, J. R., Salzer, M., Schupbach, S., Steffensen, J. P., Vinther, B. M., and Woodruff, T. E.: Timing and climate forcing of volcanic eruptions for the past 2,500 years, Nature, 523, 543-549, 2015. 
Spanish National Weather Agency - AEMet Open Data: AEMet Open Data. http://www.aemet.es/es/datos_abiertos/AEMET_OpenData, 2019.

Staudt, M., Esteban-Parra, M. J., and Castro-Díez, Y.: Homogenization of long-term monthly Spanish temperature data, International Journal of Climatology, 27, 18091823, 2007.

Steinhilber, F., Beer, J., and Fröhlich, C.: Total solar irradiance during the Holocene, Geophysical Research Letters, 36, 2009.

Trouet, V., Esper, J., Graham, N. E., Baker, A., Scourse, J. D., and Frank, D. C.: Persistent Positive North Atlantic Oscillation Mode Dominated the Medieval Climate Anomaly, Science, 324, 78-80, 2009. 